\title{
Biotypes, antibiotic resistance and plasmids coding for CFA/I and STa in enterotoxigenic Escherichia coli strains of serotype 0153: $\mathrm{H} 45$ isolated in Spain
}

\author{
E. A. GONZALEZ, J. BLANCO, I. GARABAL and M. BLANCO
} Departamento de Microbiologia y Parasitologia, Universidad de Santiago de Compostela, Facultad de Veterinaria,
27002 Lugo, Spain

\begin{abstract}
Summary. Enterotoxigenic Escherichia coli (ETEC) strains of serotype O153:H45 have been found recently to be a frequent cause of sporadic cases and outbreaks of neonatal diarrhoea in Spain and the most important cause of infant diarrhoea in Chile. Relationships between sugar fermentation patterns, resistance to antibiotics and plasmid profiles were analysed in nine $E$. coli O153:H45 strains isolated in Spain that synthesised CFA/I antigen and STa enterotoxin. Derivative strains obtained by curing with acridine orange, and transconjugants rendered antibiotic resistant, were characterised phenotypically and analysed for plasmid content. Two fermentation patterns were recognised: rhamnose fermenters (four strains) and rhamnose non-fermenters (five strains). The ability to ferment rhamnose was the only differential characteristic found among 49 carbohydrate fermentation tests used to establish fermentation patterns. All nine strains possessed similar plasmid profiles of three or four plasmids of 52-87 Mda. A non-conjugative large plasmid of $82 \mathrm{Mda}$ or $87 \mathrm{Mda}$, depending upon the strain, was identified as that responsible for production of both CFA/I and STa. Resistance to antibiotics was determined by plasmids other than those coding for CFA/I and STa. Two conjugative resistance factors were identified: a 52-Mda plasmid coding for resistance to ampicillin, streptomycin and sulphonamide in rhamnose-fermenting strains, and a 77-Mda plasmid coding for resistance to ampicillin, streptomycin, kanamycin, tetracycline and sulphonamide in rhamnose non-fermenting strains. Our results support the hypothesis that the prevalence and distribution of ETEC strains belonging to serotype O153:H45 in Spain and Chile could be due to the extensive cultural relations between Spain and South America from the past.
\end{abstract}

\section{Introduction}

Enterotoxigenic Escherichia coli (ETEC) strains are the major causative agents of diarrhoea in children in developing countries ${ }^{1-3}$ and cause occasional sporadic cases and epidemic outbreaks of diarrhoea in developed countries of diverse climate. ${ }^{4-8}$ ETEC strains are characterised according to serotype, production of thermolabile (LT) or thermostable (STa) enterotoxins, or both, and possession of fimbrial structures named colonisation factor antigens (CFAs) which enable them to attach to and proliferate in the small bowel..$^{9-12}$ Production of LT and STa enterotoxins and colonisation factor antigens CFA/I and CFA/II is determined by plasmids. ${ }^{13-16}$ Single recombinant plasmids may exist for determining production of both enterotoxin and CFA. One such plasmid determining STa and CFA/I production and one determining LT, STa and CFA/II production have been described. ${ }^{13,15,16}$ Many different serotypes have been found among ETEC strains although most strains from Asiatic and African countries are of a small number of serotypes, e.g., O6, O8, O15, $\mathrm{O} 25$ and $078 .{ }^{3}$ However, little is known about the incidence and distribution of serotypes and biotypes among ETEC strains causing sporadic cases and outbreaks of diarrhoea in South America and Europe. ${ }^{4,6,7,17,18}$ Some ETEC strains, particularly those of serotype O153:H45, have been described as the major cause of infant diarrhoea in Chile ${ }^{17}$ and were also detected occasionally in Thailand, ${ }^{19}$ the Central African Republic ${ }^{2}$ and Somalia. ${ }^{20}$

We have reported recently that ETEC strains of serotype O153:H45 producing STa and CFA/I were the most important enterotoxigenic $E$. coli strains causing sporadic cases and epidemic outbreaks of diarrhoea in children under 3 years of age in Spain. ${ }^{18}$ In this study we report the biotypes, resistance to antibiotics and plasmid profiles of these strains. Plasmids coding for STa and CFA/I were identified and the most relevant antibiotic resistance factors were localised. 


\section{Materials and methods}

\section{Bacteria}

Nine $E$. coli strains of serotype O153:H45 producing STa and CFA/I were used. They were selected from the $31 \mathrm{STa}$-producing $E$. coli strains isolated in a previous study in Spain of infantile diarrhoea. ${ }^{18}$

\section{Biotyping and sugar fermentation patterns}

Strains were biotyped by the API-20E System (API System, Montolieu Vercieu, France). Furthermore, the carbohydrate fermentation pattern of wild-type strains and cured derivates was determined with the API-50CH System. The 49 carbohydrates used as fermentation substrates were: glycerol, erythritol, Darabinose, L-arabinose, ribose, D-xylose, L-xylose, adonitol, $\beta$-methyl-D-xylose, galactose, D-glucose, $\mathrm{D}$ fructose, D-mannose, sorbose, rhamnose, dulcitol, inositol, mannitol, sorbitol, $\alpha$-methyl-D-mannoside, $\alpha$-methyl-D-glucoside, $\mathrm{N}$-acetyl-glucosamine, amygdalin, arbutine, aesculin, salicin, cellobiose, maltose, lactose, melibiose, sucrose, trehalose, inulin, melezitose, raffinose, starch, glycogen, xylitol, gentiobiose, D-turanose, D-lyxose, D-tagatose, D-fucose, L-fucose, D-arabitol, L-arabitol, gluconate, 2-ketogluconate and 5-ketogluconate. Fermentation tests were examined after incubation for $24 \mathrm{~h}$ and $48 \mathrm{~h}$ at $37^{\circ} \mathrm{C}$.

\section{Enterotoxin (STa) assay}

Production of STa was assayed by the infant mouse test (IMT) as described previously. ${ }^{21,22}$ Ratios of intestinal weight/remaining body weight $>0.1$ were considered indicative of enterotoxin production. Thermostability of STa was confirmed by re-testing culture filtrates after heating them at $80^{\circ} \mathrm{C}$ for $20 \mathrm{~min}$.

\section{Haemagglutination tests}

Mannose-resistant haemagglutinating activity (MRHA) for human erythrocytes of groups A and O, and for bovine erythrocytes was assayed by the rockedtile method described previously. ${ }^{23,24}$ It was indicative of the presence of CFA/I. ${ }^{23}$ For haemagglutination tests, bacteria were grown statically in Mueller-Hinton Broth (Difco) at $37^{\circ} \mathrm{C}$ for 5 days and the pellicle formed was plated out on casaminoacids-yeast extract agar (CFA-agar) ${ }^{25}$ and incubated for $18 \mathrm{~h}$ at $37^{\circ} \mathrm{C}$.

\section{Bacterial surface hydrophobicity determinations}

Surface hydrophobicity was measured by determining the minimal molar concentration of ammonium sulphate at which bacterial cells aggregated, according to the test described previously. ${ }^{26,27}$ Bacterial suspensions in $0.02 \mathrm{M}$ phosphate buffer $(\mathrm{pH} \mathrm{6.8)}$ were from cultures on CFA-agar incubated at $37^{\circ} \mathrm{C}$ for $18 \mathrm{~h}$ from inocula of pellicles formed in static Mueller-Hinton broth after 5 days at $37^{\circ} \mathrm{C}$.

\section{Detection of $C F A / I$}

Strains were examined for CFA/I by an improved staphylococcal co-agglutination test with Staphylococcus aureus strain Cowan I (NCTC 8530) coated with anti-CFA/I rabbit serum specifically adsorbed, as described previously. ${ }^{28}$ The specificity of the reagent for CFA/I antigen was verified. For the test, a colony of bacteria grown on CFA-agar was removed with a tooth-pick and suspended in $50 \mu \mathrm{l}$ of co-agglutination reagent without antiserum (control) and in $50 \mu$ l of coagglutination reagent with antiserum (test) on a glass slide. After rotating the slide for $30 \mathrm{~s}$, the test suspension was observed for strong agglutination which was not present in the control.

\section{Antibiotic susceptibility determinations}

Susceptibility tests were performed by the standard disk diffusion test described previously ${ }^{29}$ with (disk content): ampicillin $10 \mu \mathrm{g}$, streptomycin $10 \mu \mathrm{g}$, gentamicin $10 \mu \mathrm{g}$, kanamycin $30 \mu \mathrm{g}$, tetracycline $30 \mu \mathrm{g}$, chloramphenicol $30 \mu \mathrm{g}$, polymyxin B 300 units, nalidixic acid $30 \mu \mathrm{g}$, trimethoprim $5 \mu \mathrm{g}$, nitrofurantoin $300 \mu \mathrm{g}$ and sulphonamide $300 \mu \mathrm{g}$.

\section{Plasmid curing and conjugation experiments}

These were performed by methods described previously ${ }^{30}$ with slight modifications. In curing experiments, bacteria were grown in Luria broth (Lbroth) containing acridine orange $200 \mu \mathrm{g} / \mathrm{ml}$ for $24 \mathrm{~h}$ at $37^{\circ} \mathrm{C}$. After incubation, the appropriate dilutions of the culture were plated on CFA-agar and well-isolated colonies were tested for lack of agglutination with the specific anti-CFA/I co-agglutination reagent. Presumptive cured $\mathrm{CFA} / \mathrm{I}^{-}$colonies were re-tested for lack of agglutination after static growth for pellicle formation, and were then tested for production of STa. Conjugation experiments were performed in Lbroth by mixing $1 \mathrm{ml}$ of the donor strain culture with $10 \mathrm{ml}$ of the recipient strain culture, both previously grown in fresh $\mathrm{L}$-broth at $37^{\circ} \mathrm{C}$ for $2 \mathrm{~h}$ with shaking $(200 \mathrm{rpm})$. Conjugation matings were incubated statically for $18 \mathrm{~h}$ at $37^{\circ} \mathrm{C}$. After incubation, flasks were shaken vigorously and appropriate dilutions in sterile $\mathrm{L}$-broth were plated on $\mathrm{L}$-agar containing nalidixic acid $30 \mu \mathrm{g} / \mathrm{ml}$. Isolated $\mathrm{Nal}^{\mathrm{r}}$ colonies were subcultured into Mueller-Hinton broth incubated for 5 days at $37^{\circ} \mathrm{C}$ for pellicle formation, plated on CFA-agar for $18 \mathrm{~h}$ at $37^{\circ} \mathrm{C}$ and then tested for the presence of CFA/I by co-agglutination.

\section{DNA extraction and electrophoresis}

Plasmid DNA was extracted from cultures of bacteria according to methods published previously ${ }^{31,32}$ and electrophoresed in agarose $0.7 \%$ gels at $10 \mathrm{~V} / \mathrm{cm}$. 


\section{Control strains}

The following $E$. coli strains were used as controls: $\mathrm{H} 10407, \mathrm{~m} 452-\mathrm{Cl}$ and $\mathrm{m} 633-\mathrm{Cl}^{23}$ for production of STa and CFA/I; K12-711 lac ${ }^{-}$D-arab ${ }^{-}$rham $^{+}$nal $^{\mathrm{r}}$ as a recipient strain in conjugation experiments; $\mathrm{V} 517^{33}$ and $\mathrm{H}_{10407^{14}}$ as sources of plasmids of known size for use as markers. E. coli O153:H45 CFA/I ${ }^{+} \mathrm{STa}^{+}$ strain D100 isolated in $\mathrm{Chile}^{17}$ was used to compare its biotype and resistance to antibiotics with those shown by the other strains of this study.

\section{Results}

\section{Biotyping and fermentation patterns}

All nine $E$. coli $\mathrm{O} 153: \mathrm{H}_{45} \mathrm{STa}^{+} \mathrm{CFA} / \mathrm{I}^{+}$strains were confirmed as being $E$. coli by the API-20E system. Four strains had the profile 5144552 whereas the other five had the profile 5144542 (table I). The only difference between these two biotypes was the ability of the four strains isolated in La Coruña having the former profile to ferment rhamnose. When the $E$. coli strains were studied for their ability to ferment 49 carbohydrates, the only difference observed in their profiles was that relating to rhamnose fermentation. All nine strains fermented glycerol, D-arabinose, Larabinose, ribose, D-xylose, galactose, D-glucose, Dfructose, D-mannose, mannitol, sorbitol, $\mathrm{N}$-acetylglucosamine, maltose, lactose, melibiose, trehalose, Lfucose and gluconate. The fermentation patterns of eight other enterotoxigenic strains isolated in Spain of serotypes $\mathrm{O} 27: \mathrm{H} 7$ (three strains, $\mathrm{STa}^{+}$), O24:H15 (one strain, $\mathrm{STa}^{+}$), $\mathrm{O} 89: \mathrm{K}^{-}: \mathrm{H} 15$ (one strain, $\mathrm{STa}^{+}$) and O6:K 15:H16 (three strains $\mathrm{LT}^{+} \mathrm{STa}^{+} \mathrm{CFA} / \mathrm{II}^{+}$) were tested similarly. All of these strains fermented rhamnose but differed from the strains of serotype 0153:H45 in their fermentation patterns with at least three other sugars. To determine whether all 0153 strains isolated from each individual child exhibited the same fermentation pattern, 3-5 E. coli colonies isolated from each child were tested. All the $E$. coli colony isolates of serotype 0153: $\mathrm{H} 45$ from each child showed the same fermentation pattern whereas all the non-O153 colony isolates were of different fermentation patterns.

\section{Antibiotic susceptibility}

All nine O153:H45 strains were resistant to ampicillin, streptomycin and sulphonamide. Rhamnose non-fermenting strains in addition were resistant to kanamycin and tetracycline, whereas among the rhamnose fermenting strains, only one strain (CD68b) was resistant to kanamycin (table I).

\section{Plasmid profiles}

The plasmid profiles of all nine 0153 E. coli strains were similar and showed three or four large plasmid bands of 52-87 Mda (fig. 1). The characteristic plasmid profile for each original $\mathrm{O} 153$ strain was confirmed by the plasmid analysis of additional $\mathrm{O} 153$ colonies isolated from the same sample. All the $\mathrm{O} 153$ colonies isolated from the same child showed an identical plasmid profile. Some plasmids were common to strains which differed in biotype, isolation source and date of isolation (fig. 1).

\section{Curing of plasmids}

From a total of $587 \mathrm{E}$. coli colonies isolated after treatment of the nine 0153 strains with acridine orange investigated for lack of CFA/I antigen (table II), $64 \mathrm{CFA} / \mathrm{I}^{-}$derivative strains were investigated for the production of STa, antibiotic resistance and plasmid content. Plasmid analysis showed that CFA/I and STa were coded in a large plasmid of 82 or 87 Mda, depending on the strain (table III). Derivative strains lacking the plasmid coding for CFA/I and STa

Table I. Characteristics of E. coli O153:H45 strains producing STa and CFA/I

\begin{tabular}{|c|c|c|c|c|c|c|c|c|c|}
\hline \multirow{2}{*}{$\begin{array}{c}\text { Strain } \\
\text { no. } \\
\text { CD68b } \\
\text { CD69a } \\
\text { CD79a } \\
\text { CD99a }\end{array}$} & \multirow{2}{*}{$\begin{array}{l}\text { City of isolation* } \\
\text { La Coruña } \\
\mathrm{La} \text { Coruña } \\
\mathrm{La} \text { Coruña } \\
\mathrm{La} \text { Coruña }\end{array}$} & \multirow{2}{*}{$\begin{array}{l}\begin{array}{c}\text { Date of } \\
\text { isolation }\end{array} \\
\text { Aug. } 1986 \\
\text { Aug. } 1986 \\
\text { Aug. } 1986 \\
\text { Oct. } 1986\end{array}$} & \multirow{2}{*}{$\begin{array}{c}\begin{array}{c}\text { API-20E } \\
\text { profile }\end{array} \\
5144552 \\
5144552 \\
5144552 \\
5144552\end{array}$} & \multirow{2}{*}{$\begin{array}{c}\begin{array}{c}\text { Rhamnose } \\
\text { fermenta- } \\
\text { tion }\end{array} \\
+ \\
+ \\
+ \\
+ \\
+\end{array}$} & \multicolumn{5}{|c|}{$\begin{array}{l}\text { Antibiotic resistance } \\
\text { pattern }\end{array}$} \\
\hline & & & & & $\begin{array}{l}\text { Am } \\
\text { Am } \\
\text { Am } \\
\text { Am }\end{array}$ & $\begin{array}{l}\text { Sm } \\
\text { Sm } \\
\text { Sm } \\
\text { Sm }\end{array}$ & $\mathbf{K}$ & & $\begin{array}{l}\mathrm{Su} \\
\mathrm{Su} \\
\mathrm{Su} \\
\mathrm{Su}\end{array}$ \\
\hline $\begin{array}{l}\text { SD53a } \\
\text { SD72a }\end{array}$ & $\begin{array}{l}\text { Santiago } \\
\text { Santiago }\end{array}$ & $\begin{array}{l}\text { Oct. } 1985 \\
\text { Oct. } 1985\end{array}$ & $\begin{array}{l}5144542 \\
5144542\end{array}$ & $\begin{array}{l}- \\
-\end{array}$ & $\begin{array}{l}\mathrm{Am} \\
\mathrm{Am}\end{array}$ & $\begin{array}{l}\mathrm{Sm} \\
\mathrm{Sm}\end{array}$ & $\begin{array}{l}\mathbf{K} \\
\mathbf{K}\end{array}$ & $\begin{array}{l}\mathrm{Te} \\
\mathrm{Te}\end{array}$ & $\begin{array}{l}\text { Su } \\
\text { Su }\end{array}$ \\
\hline $\begin{array}{l}1782 \\
57767 \\
422262\end{array}$ & $\begin{array}{l}\text { Madrid } \\
\text { Madrid } \\
\text { Valencia }\end{array}$ & $\begin{array}{l}\text { Dec. } 1982 \\
\text { Oct. } 1984 \\
\text { Aug. } 1984\end{array}$ & $\begin{array}{l}5144542 \\
5144542 \\
5144542\end{array}$ & $\begin{array}{l}- \\
-\end{array}$ & $\begin{array}{l}\text { Am } \\
\text { Am } \\
\text { Am }\end{array}$ & $\begin{array}{l}\mathrm{Sm} \\
\mathrm{Sm} \\
\mathrm{Sm}\end{array}$ & $\begin{array}{l}\mathbf{K} \\
\mathbf{K} \\
\mathbf{K}\end{array}$ & $\begin{array}{l}\mathrm{Te} \\
\mathrm{Te} \\
\mathrm{Te}\end{array}$ & $\begin{array}{l}\text { Su } \\
\text { Su } \\
\text { Su }\end{array}$ \\
\hline D100 & Chile & $1982-1983$ & 5144552 & + & $\mathrm{Am}$ & $\mathrm{Sm}$ & $\mathbf{K}$ & $\mathrm{Te}$ & $\mathrm{Su}$ \\
\hline
\end{tabular}

* La Coruña and Santiago lie in north west Spain, Madrid in the central area and Valencia on the eastern coast.

Am, ampicillin; Sm, streptomycin; K, kanamycin; Te, tetracycline; Su, sulphonamide. 


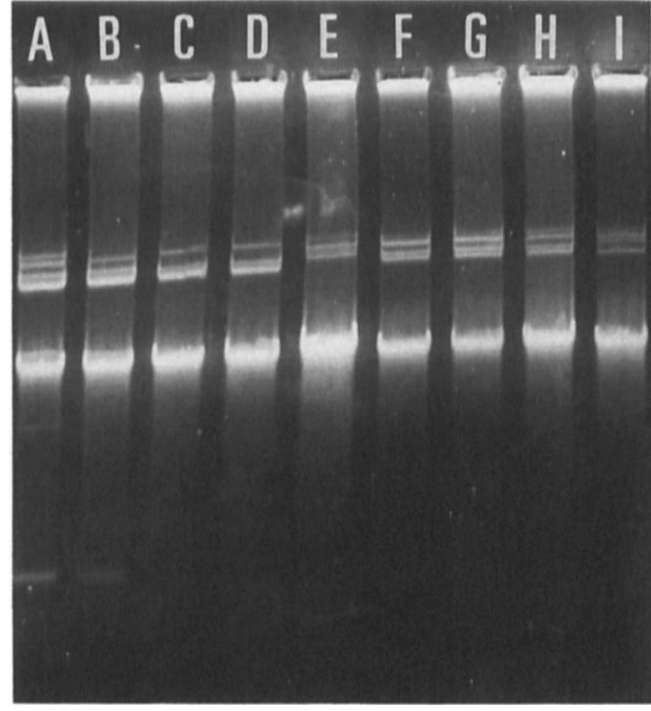

Fig. 1. Agarose gel electrophoresis of plasmid DNA from $E$. coli O153: $\mathrm{H}_{45} \mathrm{CFA} / \mathrm{I}^{+} \mathrm{STa}^{+}$strains: lane A, strain CD68b; B, strain CD69a; C, strain CD79a; D, strain CD99a; E, strain SD53a; F, strain SD72a; G, strain 1782; H, strain 57767; I, strain 422262.

usually remained resistant to antibiotics and showed the same resistance pattern as the original parent strain. Most $(55,86 \%)$ of the derivative strains lacking CFA/I were also unable to produce STa but $9(14 \%)$ derivatives from strains CD69a, CD99a and SD72a produced STa (table II). These CFA/I ${ }^{-} \mathrm{STa}^{+}$derivative strains carried a new plasmid not found in the corresponding parent strain (fig. 2). Thus, derivative strain CD69a-5 possessed a 34-Mda plasmid, CD99a2 an 82-Mda plasmid and SD72a-1 a 42-Mda plasmid. A 58-Mda plasmid not present in strain SD53a, was also detected in the $\mathrm{CFA} / \mathrm{I}^{-} \mathrm{STa}^{-}$derivative strains from this strain. Derivative strains lacking the plasmid coding for CFA/I showed lower surface hydrophobicity than their $\mathrm{CFA} / \mathrm{I}^{+}$parent strains and were MRHAnegative in tests with human and bovine erythrocytes (table III).

\section{Conjugal transfer of plasmid-borne factors}

After conjugation experiments, $450 \mathrm{Nal}^{\mathrm{r}}$ colonies were tested for production of CFA/I. No transconjugant strain producing CFA/I was found. Nevertheless, in some of the $\mathrm{Nal}^{\mathrm{r}}$ colonies the $\mathrm{lac}^{-} \mathrm{arab}^{-} \mathrm{rham}^{+}$ biotype was found. These transconjugants showed the characteristic resistance pattern of their corresponding donor strain and possessed plasmids confirming their character as transconjugant colonies. By comparing the phenotypic properties and plasmid content of

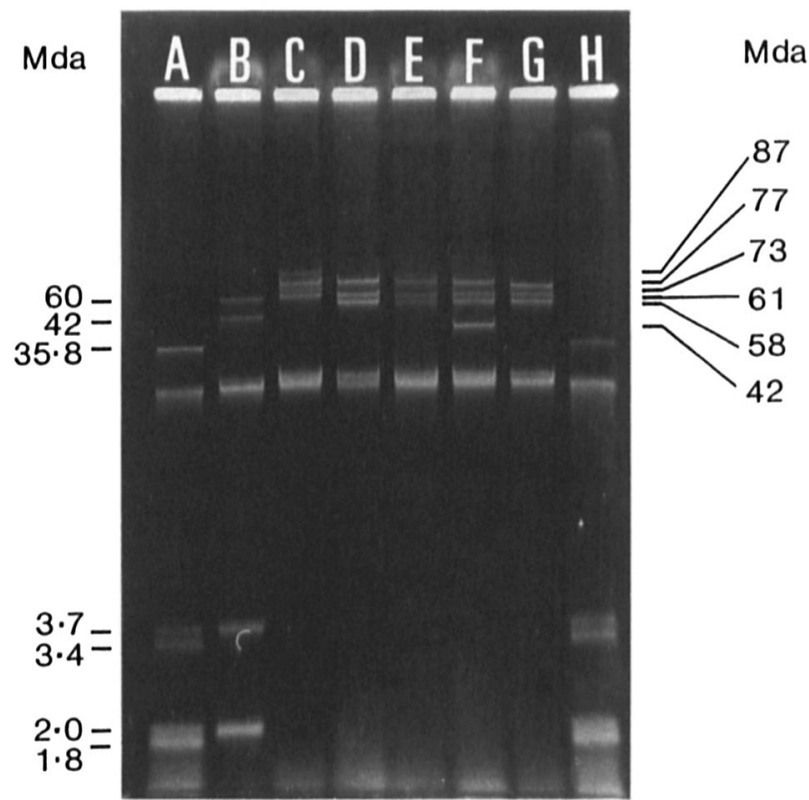

Fig. 2. Agarose gel electrophoresis of plasmid DNA from some of the original E. coli $\mathrm{Ol} 153: \mathrm{H} 45 \mathrm{CFA} / \mathrm{I}^{+} \mathrm{STa}^{+}$strains and their cured derivatives. Lanes $\mathbf{A}$ and $\mathbf{H}$, control strain V517; $\mathbf{B}$, control strain H10407; C, strain SD53a; D, derivative SD53a-2; E, strain SD72a; F, derivative SD72a-1; G, derivative SD72a-2. Numbers on the left indicate the mol. wt (Mda) of plasmids from strains V517 and H10407 used to calculate the linear regression $\left(r^{2}=0.99\right)$ of the migration rates. Numbers on the right indicate the mol. wt (Mda) of plasmids from the 0153:H45 strains and their cured derivatives.

Table II. Cured derivative colonies and transconjugants obtained from $E$. coli $\mathrm{O} 153: \mathrm{H} 45$ $\mathrm{CFA} / \mathrm{I}^{+} \mathrm{STa}^{+}$strains

\begin{tabular}{|c|c|c|c|c|c|c|}
\hline \multirow{2}{*}{$\begin{array}{l}\text { Original } \\
\text { strain }\end{array}$} & \multicolumn{4}{|c|}{ Number of derivative colonies* } & \multicolumn{2}{|c|}{$\begin{array}{l}\text { Number of transconju- } \\
\text { gants } \dagger\end{array}$} \\
\hline & tested & $\mathrm{CFA} / \mathrm{I}^{-}$ & CFA/I-STa ${ }^{-}$ & CFA/I ${ }^{-} \mathrm{STa}^{+}$ & tested & $\mathrm{CFA} / \mathrm{I}^{+} \mathrm{Nal}^{\mathrm{r}}$ \\
\hline CD68b & 120 & 1 & 1 & 0 & 50 & 0 \\
\hline CD69a & 150 & 9 & 8 & 1 & 50 & 0 \\
\hline CD79a & 120 & 8 & 8 & 0 & 50 & 0 \\
\hline CD99a & 74 & 9 & 6 & 3 & 50 & 0 \\
\hline SD53a & 60 & 5 & 5 & 0 & 50 & 0 \\
\hline SD72a & 33 & 7 & 2 & 5 & 50 & 0 \\
\hline 1782 & 10 & 10 & 10 & 0 & 50 & 0 \\
\hline 57767 & 10 & 10 & 10 & 0 & 50 & 0 \\
\hline 422262 & 10 & 5 & 5 & 0 & 50 & 0 \\
\hline
\end{tabular}

* Obtained by growing bacteria in the presence of acridine orange.

$\dagger$ With $E$. coli K12-711 $\mathrm{Nal}^{\mathrm{r}}$ as recipient. 
Table III. Phenotypes and plasmid profiles of cured derivatives and transconjugants from E. coli O153:H45 STa ${ }^{+}$CFA/I ${ }^{+}$ strains isolated in Spain

\begin{tabular}{|c|c|c|c|c|c|c|c|c|c|c|c|c|}
\hline \multirow{3}{*}{$\begin{array}{c}\begin{array}{c}\text { Strain* } \\
\text { no. }\end{array} \\
\text { CD68b }\end{array}$} & \multirow{3}{*}{$\begin{array}{l}\text { Biotype** }^{* *} \\
\text { Arab }^{+} \text {Rham }^{+}\end{array}$} & \multicolumn{2}{|c|}{ Production of } & \multirow{3}{*}{$\begin{array}{c}\text { Hydrophobicity } \ddagger \\
0.06\end{array}$} & \multirow{2}{*}{ MRHA§ } & \multirow{2}{*}{\multicolumn{5}{|c|}{ Resistance pattern $\|$}} & \multirow{2}{*}{\multicolumn{2}{|c|}{ Plasmid profileף }} \\
\hline & & CFA/I & STa $†$ & & & & & & & & & \\
\hline & & + & + & & + & $\mathrm{Am}$ & $\mathrm{Sm}$ & $\mathbf{K}$ & & $\mathrm{Su}$ & & $87 ; 82 ; 65 ; 52 ; 13 ; 3 \cdot 4$ \\
\hline CD68b-1 & $\mathrm{Lac}^{+} \mathrm{Arab}^{+} \mathrm{Rham}^{+}$ & - & - & 1.00 & - & Am & $\mathrm{Sm}$ & $\mathbf{K}$ & & $\mathrm{Su}$ & & $87 ; 65 ; 52 ; 13 ; 3.4 ; 2.1$ \\
\hline $\begin{array}{l}\text { CD69a } \\
\text { CD69a-5 } \\
\text { CD69a-6 } \\
\text { T-CD69a-a }\end{array}$ & $\begin{array}{l}\mathrm{Lac}^{+} \mathrm{Arab}^{+} \mathrm{Rham}^{+} \\
\mathrm{Lac}^{+} \mathrm{Arab}^{+} \mathrm{Rham}^{+} \\
\mathrm{Lac}^{+} \mathrm{Arab}^{+} \mathrm{Rham}^{+} \\
\mathrm{Lac}^{-} \mathrm{Arab}^{-} \mathrm{Rham}^{+}\end{array}$ & $\begin{array}{l}+ \\
- \\
- \\
-\end{array}$ & $\begin{array}{l}+ \\
+ \\
- \\
-\end{array}$ & $\begin{array}{l}0.06 \\
1.00 \\
1.00 \\
1.00\end{array}$ & $\begin{array}{l}+ \\
- \\
- \\
-\end{array}$ & $\begin{array}{l}\mathrm{Am} \\
\mathrm{Am} \\
\mathrm{Am} \\
\mathrm{Am}\end{array}$ & $\begin{array}{l}\mathrm{Sm} \\
\mathrm{Sm} \\
\mathrm{Sm} \\
\mathrm{Sm}\end{array}$ & & & $\begin{array}{l}\mathrm{Su} \\
\mathrm{Su} \\
\mathrm{Su} \\
\mathrm{Su}\end{array}$ & $\mathbf{N a}$ & $\begin{array}{c}82 ; 65 ; 52 ; 3 \cdot 4 ; 2 \cdot 1 \\
65 ; 52 ; 34 ; 3 \cdot 4 ; 2 \cdot 1 \\
65 ; 52 ; 3 \cdot 4 ; 2 \cdot 1 \\
52\end{array}$ \\
\hline $\begin{array}{l}\text { CD79a } \\
\text { CD79a-1 } \\
\text { CD79a-2 }\end{array}$ & $\begin{array}{l}\mathrm{Lac}^{+} \mathrm{Arab}^{+} \mathrm{Rham}^{+} \\
\mathrm{Lac}^{+} \mathrm{Arab}^{+} \mathrm{Rham}^{+} \\
\mathrm{Lac}^{+} \mathrm{Arab}^{+} \mathrm{Rham}^{+}\end{array}$ & $\begin{array}{l}+ \\
- \\
-\end{array}$ & $\begin{array}{l}+ \\
- \\
-\end{array}$ & $\begin{array}{l}0.06 \\
1.00 \\
1.00\end{array}$ & $\begin{array}{l}+ \\
- \\
-\end{array}$ & $\begin{array}{l}\mathrm{Am} \\
\mathrm{Am} \\
\mathrm{Am}\end{array}$ & $\begin{array}{l}\mathrm{Sm} \\
\mathrm{Sm} \\
\mathrm{Sm}\end{array}$ & & & $\begin{array}{l}\mathrm{Su} \\
\mathrm{Su} \\
\mathrm{Su}\end{array}$ & & $\begin{array}{c}87 ; 65 ; 52 \\
52 \\
65 ; 52\end{array}$ \\
\hline $\begin{array}{l}\text { CD99a } \\
\text { CD99a-1 } \\
\text { CD99a-2 } \\
\text { CD99a-6 }\end{array}$ & $\begin{array}{l}\mathrm{Lac}^{+} \mathrm{Arab}^{+} \mathrm{Rham}^{+} \\
\mathrm{Lac}^{+} \mathrm{Arab}^{+} \text {Rham }^{+} \\
\mathrm{Lac}^{+} \mathrm{Arab}^{+} \text {Rham } \\
\mathrm{Lac}^{+} \mathrm{Arab}^{+} \text {Rham }\end{array}$ & $\begin{array}{l}+ \\
- \\
- \\
-\end{array}$ & $\begin{array}{l}+ \\
- \\
+ \\
+\end{array}$ & $\begin{array}{l}0.06 \\
1 \cdot 00 \\
1 \cdot 00 \\
1 \cdot 00\end{array}$ & $\begin{array}{l}+ \\
- \\
- \\
-\end{array}$ & $\begin{array}{l}\mathrm{Am} \\
\mathrm{Am}\end{array}$ & $\begin{array}{l}\mathrm{Sm} \\
\mathrm{Sm} \\
\mathrm{Sm}\end{array}$ & & & $\begin{array}{l}\mathrm{Su} \\
\mathrm{Su} \\
\mathrm{Su} \\
\mathrm{Su}\end{array}$ & & $\begin{array}{c}87 ; 65 ; 52 \\
65 ; 52 \\
82 ; 65 ; 52 \\
82 ; 65\end{array}$ \\
\hline $\begin{array}{l}\text { SD53a } \\
\text { SD53a-2 } \\
\text { SD53a-5 } \\
\text { T-SD53a-d }\end{array}$ & $\begin{array}{l}\mathrm{Lac}^{+} \mathrm{Arab}^{+} \mathrm{Rham}^{-} \\
\mathrm{Lac}^{+} \mathrm{Arab}^{+} \mathrm{Rham}^{-} \\
\mathrm{Lac}^{+} \mathrm{Arab}^{+} \mathrm{Rham}^{-} \\
\mathrm{Lac}^{-} \mathrm{Arab}^{-} \text {Rham }^{+}\end{array}$ & $\begin{array}{l}+ \\
- \\
-\end{array}$ & $\begin{array}{l}+ \\
- \\
- \\
-\end{array}$ & $\begin{array}{l}0.06 \\
1.00 \\
0.40 \\
1.00\end{array}$ & $\begin{array}{l}+ \\
- \\
- \\
-\end{array}$ & $\begin{array}{l}\text { Am } \\
\text { Am } \\
\text { Am }\end{array}$ & $\begin{array}{l}\mathrm{Sm} \\
\mathrm{Sm} \\
\mathrm{Sm} \\
\mathrm{Sm}\end{array}$ & $\begin{array}{l}\mathbf{K} \\
\mathbf{K} \\
\mathbf{K} \\
\mathbf{K}\end{array}$ & $\begin{array}{l}\mathrm{Te} \\
\mathrm{Te} \\
\mathrm{Te} \\
\mathrm{Te}\end{array}$ & $\begin{array}{l}\mathrm{Su} \\
\mathrm{Su} \\
\mathrm{Su} \\
\mathrm{Su}\end{array}$ & $\mathrm{Na}$ & $\begin{array}{c}87 ; 77,73,61 \\
77 ; 61 ; 58 \\
77 ; 61 ; 58 \\
77 ; 58\end{array}$ \\
\hline $\begin{array}{l}\text { SD72a } \\
\text { SD72a-1 } \\
\text { SD72a-2 }\end{array}$ & $\begin{array}{l}\mathrm{Lac}^{+} \mathrm{Arab}^{+} \mathrm{Rham}^{-} \\
\mathrm{Lac}^{+} \mathrm{Arab}^{+} \mathrm{Rham}^{-} \\
\mathrm{Lac}^{+} \mathrm{Arab}^{+} \mathrm{Rham}^{-}\end{array}$ & $\begin{array}{l}+ \\
- \\
-\end{array}$ & $\begin{array}{l}+ \\
+ \\
-\end{array}$ & $\begin{array}{l}0.06 \\
1.00 \\
1.00\end{array}$ & $\begin{array}{l}+ \\
- \\
-\end{array}$ & $\begin{array}{l}\mathrm{Am} \\
\mathrm{Am} \\
\mathrm{Am}\end{array}$ & $\begin{array}{l}\mathrm{Sm} \\
\mathrm{Sm} \\
\mathrm{Sm}\end{array}$ & $\begin{array}{l}\mathbf{K} \\
\mathbf{K}\end{array}$ & $\begin{array}{l}\mathrm{Te} \\
\mathrm{Te} \\
\mathrm{Te}\end{array}$ & $\begin{array}{l}\text { Su } \\
\text { Su } \\
\text { Su }\end{array}$ & & $\begin{array}{c}87 ; 77 ; 61 ; 58 \\
77 ; 61 ; 58 ; 42 \\
77 ; 61 ; 58\end{array}$ \\
\hline $\begin{array}{l}1782 \\
1782-1 \\
1782-2 \\
T-1782-a\end{array}$ & $\begin{array}{l}\mathrm{Lac}^{+} \mathrm{Arab}^{+} \mathrm{Rham}^{-} \\
\mathrm{Lac}^{+} \mathrm{Arab}^{+} \mathrm{Rham}^{-} \\
\mathrm{Lac}^{+} \mathrm{Arab}^{+} \mathrm{Rham}^{-} \\
\mathrm{Lac}^{-} \mathrm{Arab}^{-} \mathrm{Rham}^{+}\end{array}$ & $\begin{array}{l}+ \\
- \\
- \\
-\end{array}$ & $\begin{array}{l}+ \\
- \\
-\end{array}$ & $\begin{array}{l}0.06 \\
0.40 \\
1.00 \\
1.00\end{array}$ & $\begin{array}{l}+ \\
- \\
- \\
-\end{array}$ & $\begin{array}{l}\text { Am } \\
\text { Am } \\
\text { Am }\end{array}$ & $\begin{array}{l}\mathrm{Sm} \\
\mathrm{Sm} \\
\mathrm{Sm}\end{array}$ & $\begin{array}{l}\mathbf{K} \\
\mathbf{K}\end{array}$ & $\begin{array}{l}\mathrm{Te} \\
\mathrm{Te} \\
\mathrm{Te}\end{array}$ & $\begin{array}{l}\mathrm{Su} \\
\mathrm{Su} \\
\mathrm{Su}\end{array}$ & $\mathrm{Na}$ & $\begin{array}{c}87 ; 77 ; 61 ; 58 \\
61 ; 58 \\
77 ; 61 ; 58 \\
77\end{array}$ \\
\hline $\begin{array}{l}57767 \\
57767-1 \\
\text { T-57767-b } \\
\text { T-57767-c }\end{array}$ & $\begin{array}{l}\mathrm{Lac}^{+} \mathrm{Arab}^{+} \mathrm{Rham}^{-} \\
\mathrm{Lac}^{+} \mathrm{Arab}^{+} \mathrm{Rham}^{-} \\
\mathrm{Lac}^{-} \mathrm{Arab}^{-} \text {Rham }^{+} \\
\mathrm{Lac}^{-} \mathrm{Arab}^{-} \text {Rham }^{+}\end{array}$ & $\begin{array}{l}+ \\
- \\
- \\
-\end{array}$ & $\begin{array}{l}+ \\
- \\
- \\
-\end{array}$ & $\begin{array}{l}0.06 \\
0.40 \\
1.00 \\
1.00\end{array}$ & $\begin{array}{l}+ \\
- \\
- \\
-\end{array}$ & $\begin{array}{l}\mathrm{Am} \\
\mathrm{Am} \\
\mathrm{Am} \\
\mathrm{Am}\end{array}$ & $\begin{array}{l}\mathrm{Sm} \\
\mathrm{Sm} \\
\mathrm{Sm} \\
\mathrm{Sm}\end{array}$ & $\begin{array}{l}\mathbf{K} \\
\mathbf{K} \\
\mathbf{K} \\
\mathbf{K}\end{array}$ & $\begin{array}{l}\mathrm{Te} \\
\mathrm{Te} \\
\mathrm{Te} \\
\mathrm{Te}\end{array}$ & $\begin{array}{l}\mathrm{Su} \\
\mathrm{Su} \\
\mathrm{Su} \\
\mathrm{Su}\end{array}$ & $\begin{array}{l}\mathrm{Na} \\
\mathrm{Na}\end{array}$ & $\begin{array}{c}87 ; 77 ; 61 ; 58 \\
77 ; 61 ; 58 \\
77 ; 61 \\
77\end{array}$ \\
\hline $\begin{array}{l}422262 \\
422262-4 \\
422262-5 \\
T-422262-a\end{array}$ & $\begin{array}{l}\mathrm{Lac}^{+} \mathrm{Arab}^{+} \mathrm{Rham}^{-} \\
\mathrm{Lac}^{+} \mathrm{Arab}^{+} \mathrm{Rham}^{-} \\
\mathrm{Lac}^{+} \mathrm{Arab}^{+} \mathrm{Rham}^{-} \\
\mathrm{Lac}^{-} \mathrm{Arab}^{-} \text {Rham }^{+}\end{array}$ & $\begin{array}{l}+ \\
- \\
- \\
-\end{array}$ & $\begin{array}{l}+ \\
- \\
- \\
-\end{array}$ & $\begin{array}{l}0.06 \\
1.00 \\
1.00 \\
1.00\end{array}$ & $\begin{array}{l}+ \\
- \\
- \\
-\end{array}$ & $\begin{array}{l}\mathrm{Am} \\
\mathrm{Am} \\
\mathrm{Am}\end{array}$ & $\begin{array}{l}\mathrm{Sm} \\
\mathrm{Sm} \\
\mathrm{Sm} \\
\mathrm{Sm}\end{array}$ & $\begin{array}{l}\mathbf{K} \\
\mathbf{K} \\
\mathbf{K} \\
\mathbf{K}\end{array}$ & $\begin{array}{l}\mathrm{Te} \\
\mathrm{Te} \\
\mathrm{Te} \\
\mathrm{Te}\end{array}$ & $\begin{array}{l}\mathrm{Su} \\
\mathrm{Su} \\
\mathrm{Su} \\
\mathrm{Su}\end{array}$ & $\mathrm{Na}$ & $\begin{array}{c}87 ; 77 ; 73 ; 58 \\
77 ; 73 \\
77 ; 73 ; 58 \\
77 ; 58\end{array}$ \\
\hline
\end{tabular}

* Cured derivative strains $(-1,-2, \ldots)$ and transconjugants (T-) are indicated for each original strain.

** Fermentation $(+)$ or lack of fermentation $(-)$ of lactose (Lac), arabinose (Arab) and rhamnose (Rham).

$\dagger$ Infant mouse enterotoxin test. STa producers $(+)$ yielded ratios of $0.121-0.147$, whereas Sta $^{-}$strains $(-)$yielded ratios of 0.058-0.073.

$\ddagger$ Minimal molar ammonium sulphate concentration in which bacterial cells aggregated.

$\S$ With human group $A$ and $O$ erythrocytes and bovine erythrocytes.

II See footnote \& table I; Na, nalidixic acid.

T Mol. wt of plasmids is expressed in Mda. Plasmids coding for production of CFA/I and STa are in bold.

these transconjugant strains it was possible to identify the plasmids coding for antibiotic resistance in the original O153 strains (table III). Two resistance factors transferable by conjugation were detected: a $52-\mathrm{Mda}$ plasmid found in transconjugants from rhamnosefermenting strains coding for resistance to ampicillin, streptomycin and sulphonamide, and a 77-Mda plasmid found in transconjugants from rhamnose-nonfermenting strains coding for resistance to ampicillin, streptomycin, kanamycin, tetracycline and sulphonamide. Transconjugant colonies that had received antibiotic resistance factors showed the same API$20 \mathrm{E}$ and $\mathrm{API}-50 \mathrm{CH}$ profiles as the recipient strain K12-711, and also showed bacterial surface hydrophobicity similar to that of the recipient strain.

\section{Discussion}

E. coli strains CD68b, CD69a, CD79a and CD99a of serotype $\mathrm{O} 153: \mathrm{H} 45$, isolated from sporadic cases of diarrhoea in La Coruña between August and October 1986, were all rhamnose fermenters and, except for resistance to kanamycin in strain CD68b, showed the same pattern of resistance to antibiotics. These results indicate that the "apparent" sporadic cases of diarrhoea associated with these strains must be assumed to represent an unclassified outbreak caused by a single $\mathrm{O} 153$ epidemic strain. Nevertheless, although plasmid profiles for these four strains were very similar, they were not identical and the plasmid profiles of strains CD68b and CD69a were slightly 
different from those of strains CD79a and CD99a, supporting the view that these four $\mathrm{O} 153$ strains were not of the same bacterial clone (fig. 1). On the other hand, $E$. coli strains SD53a and SD72a of serotype O153:H45 isolated in Santiago, $70 \mathrm{~km}$ from La Coruña, were rhamnose non-fermenters and showed plasmid profiles and antibiotic-resistance patterns considerably different from those of the strains isolated in La Coruña. Furthermore, E. coli strains 1782, 57767 and 422262 of serotype O153:H45 associated with outbreaks of diarrhoea in Madrid and Valencia in 1982 and 1984, showed a very similar plasmid profile; all were rhamnose non-fermenters and possessed the same antibiotic-resistance pattern as the Santiago isolates (table III).

Many different bio-serotypes have been described for ETEC strains ${ }^{1,3}$ although little is known about the distribution of strains and biotypes of the ETEC serotype O153:H45. Such strains have been detected in the Central African Republic, ${ }^{2}$ Thailand ${ }^{1}$ and Somalia, ${ }^{20}$ and were found to be the most common enterotoxigenic strains causing infantile diarrhoea in Chile ${ }^{17,34}$ and Spain. ${ }^{7,18}$ This latter finding suggests that the selection and distribution of this ETEC clone could have been influenced by the close cultural connections from the past between Spain and South America. Our results may support this hypothesis on the basis of the similarities between the biotypes, resistances to antibiotics and plasmid profiles in strains isolated in Spain and those described for strains isolated in Chile. ${ }^{17}$ Nevertheless, we have found some differences in biotypes and resistance patterns. Thus, among the 0153 strains isolated in Spain we have found two biotypes based on the ability to ferment rhamnose (table I). This result suggests the existence in Spain of at least two clones of O153:H45 strains producing CFA/I and STa. The Chilean strain D100 ${ }^{17}$ was of the rhamnose-fermenting biotype and showed a fermentation pattern for the other sugars identical to that found for the strains isolated in Spain. Thus, O153 strains of the rhamnose-fermenting biotype were present in La Coruña and Chile, whereas strains of the rhamnose-non-fermenting biotype were distributed widely inside Spain (table I) having been isolated in Santiago, Madrid and Valencia. We have no information about the complete biotypes of ETEC O153:H45 strains isolated in countries other than Spain and Chile, but it would be interesting to analyse these strains at least for the fermentation of rhamnose, their antibiotic resistance patterns and plasmid profiles.

Genes coding for CFA/I and STa in O153 strains have been found previously to be localised in one plasmid of $52-78 \mathrm{Mda}$ in strains from Chile, ${ }^{17}$ or of 60 Mda in a strain from the Central African Republic. ${ }^{2}$ For the O153 strains isolated in Spain, we have found these plasmids to be 82 and $87 \mathrm{Mda}$, and, as found by others $^{34}$ working with Chilean strains, to be nonconjugative.

All the Spanish O153 strains possessed resistance factors transferable at high frequency by conjugation to $E$. coli K12-711 (table III), and, like those of the O153 strains isolated in Chile, Somalia, Thailand and the Central African Republic, ${ }^{2,17,20,34}$ they were resistant to ampicillin, streptomycin and sulphonamide. The Spanish strains of the rhamnose-non-fermenting biotype were also resistant to kanamycin and tetracycline, like the 0153 strains isolated in the countries cited above. Thus, the ETEC 0153 strains isolated in different countries have a similar pattern of resistance to antibiotics. We have found that plasmids coding for CFA/I and STa and those coding for resistance to antibiotics remain as separate entities in bacteria of serotype O153:H45. Although the existence of recombinant plasmids coding together for enterotoxins, colonisation factors and resistance to antibiotics, has been described, ${ }^{10,13,16}$ ETEC strains are usually less frequently multi-resistant to antibiotics than non-ETEC strains, although they may carry more plasmids than non-ETEC strains. This suggests the existence of a generalised process of incompatibility between enterotoxigenic plasmids and drug resistance factors as has been reported previously..$^{35}$

Some of the derivative strains obtained after curing with acridine orange contained plasmids of a size not found in the original strains from which they were obtained (table III). Some such strains, e.g., CD69a-5, CD99a-2, CD99a-6 and SD72a-1, were unable to produce CFA/I but produced STa. We speculate that the plasmids unique to these atypical derivatives arose through the dissociation of the recombinant plasmid coding for both CFA/I and STa. Additional experiments will be required to confirm this hypothesis.

Plasmids present in strains CD69a, SD53a, 1782 and 422262 were digested with restriction endonucleases $E c o$ RI and BamHI. Restriction fragment patterns obtained in agarose $1.5 \%$ gels showed extensive, but not total, homology between the plasmids contained in these four O153 strains (data not shown).

In conclusion, our results suggest that ETEC O153:H45 strains producing CFA/I and STa may be considered endemic, widespread enteropathogens in Spain and that many of the sporadic cases and outbreaks of infantile diarrhoea in this country are caused by rhamnose-fermenting or non-fermenting clones of this serotype. Moreover, Spanish and Chilean O153 ETEC strains possessed many characteristics in common, supporting previous observations. ${ }^{7}$ This may indicate the spread of these organisms from a common origin to these countries because of past extensive cultural relationships between them.

This study was supported by grants (XUGA 8430489 and 84301188) from the Xunta de Galicia (Autonomous Government of Galicia, Spain), F.I.Sss 90/0447-2) from Fondo de Investigaciones Sanitarias de la Seguridad Social, and (DGICYT PM89-0142) from Dirección General de Investigación Cientifica y Técnica. JIG and MJ acknowledge the Ministerio de Educación y Ciencia (Spain) for F.P.I. research fellowships. We thank S. Fernández for skilful technical assistance. 


\section{References}

1. Echeverria P, Ørskov F, Ørskov I, Plianbangchang D. Serotypes of enterotoxigenic Escherichia coli in Thailand and the Philippines. Infect Immun 1982; 36: 851-856.

2. Georges MC, Wachsmuth IK, Bikness KA, Moseley SL, Georges AJ. Genetic probes for enterotoxigenic Escherichia coli isolated from childhood diarrhea in the Central African Republic. J Clin Microbiol 1983; 18: 199-202.

3. Ørskov I, Ørskov F. Special O:K:H serotypes among enterotoxigenic $E$. coli strains from diarrhea in adults and children. Med Microbiol Immunol 1977: 163: 99-110.

4. Blanco J, González EA, Bernárdez I, Varela BR. Enterotoxigenic and enteropathogenic Escherichia coli in Galicia (north-west Spain). Med Microbiol Immunol 1983; 172: 165-169.

5. Brunton J, Hinde D, Langston C, Gross R, Rowe B, Gurwith M. Enterotoxigenic Escherichia coli in Central Canada. $J$ Clin Microbiol 1980; 11 : 343-348.

6. Danielsson M-L, Möllby R, Brag $\mathrm{H}$ et al. Enterotoxigenic enteric bacteria in foods and outbreaks of food-borne diseases in Sweden. J Hyg 1979; 83: 33-40.

7. Escribano A, Ørskov I, Ørskov F, Borras R. Enterotoxigenic Escherichia coli O153: $\mathrm{H} 45$ from an outbreak of diarrhoea in Spain. Med Microbiol Immunol 1987; 176: 241-244.

8. Riordan T, Gross RJ, Rowe B, Scotland SM, Johnston SM. An outbreak of food-borne enterotoxigenic Escherichia coli diarrhoea in England. $J$ Infect $1985 ; 11$ : 167-171.

9. Evans DG, Evans DJ. New surface-associated heat-labile colonization factor antigen (CFA/II) produced by enterotoxigenic Escherichia coli of serogroups $\mathrm{O6}$ and $\mathrm{O8}$. Infect Immun 1978; 21 : 638-647.

10. Gaastra W, de Graaf FK. Host-specific fimbrial adhesins of noninvasive enterotoxigenic Escherichia coli strains. Microbiol Rev 1982; 46: 129-161.

11. Levine MM, Ristaino P, Sack RB, Kaper JB, Ørskov F, Ørskoy I. Colonization factor antigens I and II and type 1 somatic pili in enterotoxigenic Escherichia coli: relation to enterotoxin type. Infect Immun 1983; 39: 889-897.

12. Levine MM. Escherichia coli that cause diarrhea: enterotoxigenic, enteropathogenic, enteroinvasive, enterohemorrhagic, and enteroadherent. $J$ Infect Dis 1987; 155: 377389.

13. Elwell LP, Shipley PL. Plasmid-mediated factors associated with virulence of bacteria to animals. Ann Rev Microbiol $1980 ; 34: 465-496$.

14. Evans DG, Silver RP, Evans DJ, Chase DG, Gorbach SL. Plasmid-controlled colonization factor associated with virulence in Escherichia coli enterotoxigenic for humans. Infect Immun 1975; 12 : 656-667.

15. Smith HR, Cravioto A, Willshaw GA et al. A plasmid coding for the production of colonization factor antigen-I and heat-stable enterotoxin in strains of Escherichia coli of serogroup 078. FEMS Microbiol Lett 1979; 6: 225-260.

16. Willshaw GA, Smith HR McConnell MM, Barclay EA Krnjulac J, Rowe B. Genetic and molecular studies of plasmids coding for colonization factor antigen I and heatstable enterotoxin in several Escherichia coli serotypes. Infect Immun 1982; 37: 858-868.

17. Agüero ME, Reyes L, Prado V, Ørskov I, Ørskov F, Cabello FC. Enterotoxigenic Escherichia coli in a population of infants with diarrhea in Chile. J Clin Microbiol 1985; 22: 576-581.

18. Blanco J, González EA, Blanco $M$ et al. Prevalence of enterotoxigenic Escherichia coli strains in outbreaks and sporadic cases of diarrhoea in Spain. Eur J Clin Microbiol Infect Dis 1989; 8: 396-400.
19. Echeverria P, Seriwatana J, Taylor DN et al. Plasmids coding for colonization factor antigens I and II, heat-labile enterotoxin, and heat-stable enterotoxin A2 in Escherichia coli. Infect Immun 1986; 51: 626-630.

20. Caprioli A, Falbo V, Ruggeri FM, Bisicchia R, Casalino M, Donelli G. Comparison among enterotoxigenic strains of Escherichia coli isolated in Italy and Somalia. Eur $J$ Epidemiol 1988; 3: 251-255.

21. Dean AG, Ching Y-C, Williams RG, Harden LB. Test for Escherichia coli enterotoxin using infant mice: application in a study of diarrhea in children in Honolulu. $J$ Infect Dis 1972: 125: 407-411.

22. González EA, Blanco J. Comparison between enterotoxigenic activity and methanol solubility in heat-stable enterotoxins (STa and STb) from Escherichia coli of human, porcine and bovine origins. Zbl Bakt Hyg 1985; A260: 1-7.

23. Blanco, J, González EA, Anadón R. Colonization antigens and haemagglutination patterns of human Escherichia coli. Eur J Clin Microbiol 1985; 4: 316-326.

24. González EA, Blanco J. Comparative study of inhibition of mannose-resistant hemagglutination caused by CFA/I CFA/II, K88-positive and K99-positive Escherichia coli strains. FEMS Microbiol Lett 1985; 29: 115-121.

25. Evans DG, Evans DJ, Tjoa W. Hemagglutination of human group A erythrocytes by enterotoxigenic Escherichia coli isolated from adults with diarrhea: correlation with colonization factor. Infect Immun 1977; 18: 330-337.

26. González EA, Blanco J, Baloda SB, Wadström T. Relative cell surface hydrophobicity of Escherichia coli strains with various recognized fimbrial antigens and without recognized fimbriae. Zbl Bakt Hyg 1988; A269: 218-236.

27. Rozgonyi F, Szitha KR, Ljungh A, Baloda SB, Hjertén S, Wadström $T$. Improvement of the salt aggregation test to study bacterial cell-surface hydrophobicity. FEMS Microbiol Lett 1985; 30: 131-138.

28. Kronvall G. A rapid slide-agglutination method for typing pneumococci by means of specific antibody adsorbed to protein A-containing staphylococci. J Med Microbiol 1973; 6: $187-190$.

29. Barry AL, Thornsberry C. Susceptibility testing: diffusion test procedures. In: Lennette EH, Balows A, Hausler WJ, Truant JP (eds) Manual of clinical microbiology, 3rd edn Washington, DC, American Society for Microbiology. 1980: 463-474.

30. Caro L, Churchward G, Chandler M. Study of plasmid replication in vivo. In: Bennett PM, Grinsted J (eds) Methods in microbiology, vol 17. London, Academic Press. 1984: 97-122.

31. Kado CI, Liu ST. Rapid procedure for detection and isolation of large and small plasmids. J Bacteriol 1981; 145: 13651373.

32. Birnboim HC, Doly J. A rapid alkaline extraction procedure for screening recombinant plasmid DNA. Nucleic Acids Res 1979; 7: 1513-1523.

33. Macrina FL, Kopecko DJ, Jones KR, Ayers DJ, McCowen SM. A multiple plasmid-containing Escherichia coli strain convenient source of size reference plasmid molecules. Plasmid 1978; 1: 417-420.

34. Fernández-Beroš ME, Kissel V, Agüero ME et al. Further characterization of Escherichia coli O153: H45, an ETEC serotype disseminated in Chile. Can J Microbiol 1988; 34: 85-88.

35. González EA, Blanco J. Relation between antibiotic resistance and number of plasmids in enterotoxigenic and nonenterotoxigenic Escherichia coli strains. Med Microbiol Immunol 1985; 174: 257-265. 